\title{
Identification of key candidate genes and pathways in oral squamous cell carcinoma by integrated Bioinformatics analysis
}

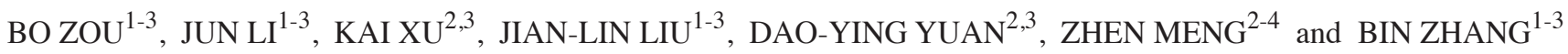 \\ ${ }^{1}$ Department of Oral Maxillofacial Surgery, School of Stomatology, Shandong University, Jinan, Shandong 250100; \\ ${ }^{2}$ Key Laboratory of Oral Maxillofacial-Head and Neck Medical Biology of Shandong Province, Liaocheng People's Hospital; \\ ${ }^{3}$ Department of Oral and Maxillofacial Surgery, Liaocheng People's Hospital, Medical College of Liaocheng University; \\ ${ }^{4}$ Precision Biomedical Key Laboratory, Liaocheng People's Hospital, Liaocheng, Shandong 252000, P.R. China
}

Received June 25, 2018; Accepted February 15, 2019

DOI: $10.3892 / \mathrm{etm} .2019 .7442$

\begin{abstract}
Oral squamous cell carcinoma (OSCC) is one of the most common types of malignant head and neck tumor, which poses a serious threat to human health. In recent years, the incidence of OSCC has been increasing, while the prognosis has not significantly improved. Elucidation of the molecular mechanisms underlying the development of OSCC may provide novel therapeutic strategies. In the present study, the gene expression profiles from 4 datasets, including 244 OSCC and 95 normal oral mucosa samples, were subjected to statistical and Bioinformatics analysis. A total of 34 differentially expressed genes (DEGs) were identified, among which 14 were upregulated and 20 were downregulated in OSCC compared with normal oral mucosa tissues. Gene Ontology enrichment analysis indicated that the DEGs were mainly involved in regulation of the immune response, cell adhesion and cell proliferative processes. The Kyoto Encyclopedia of Genes and Genomes pathway analysis revealed that the DEGs were mainly associated with the phosphoinositide- 3 kinase Akt and Toll-like receptor signaling pathway. The key candidate DEGs were identified from the complex protein-protein interaction network, and secreted phosphoprotein 1 (SPP1), integrin subunit $\alpha 3$ and plasminogen activator, urokinase (PLAU) were confirmed to be significantly associated with the survival rate. Cell Counting Kit-8 and Transwell assays demonstrated that SPP1 and PLAU regulate cell proliferation, migration and invasion. The candidate genes/pathways identified in the
\end{abstract}

Correspondence to: Dr Bin Zhang, Department of Oral and Maxillofacial Surgery, Liaocheng People's Hospital, Medical College of Liaocheng University, 67 Dongchangxi Road, Liaocheng, Shandong 252000, P.R. China

E-mail: zhangbinlc@sdu.edu.cn

Dr Zhen Meng, Precision Biomedical Key Laboratory, Liaocheng People's Hospital, 67 Dongchangxi Road, Liaocheng, Shandong 252000, P.R. China

E-mail: chinamengzhen@sdu.edu.cn

Key words: oral squamous cell carcinoma, bioinformatical analysis, differentially expressed genes, signaling pathway present study may include promising diagnostic biomarkers or therapeutic targets for OSCC.

\section{Introduction}

Oral squamous cell carcinoma (OSCC) is one of the most common types of malignancy of the head and neck, and $>500,000$ new cases are diagnosed with OSCC each year (1). The improvement of the clinical therapeutic methods has not brought any marked parallel improvement of the prognosis of OSCC, as the 5-year overall survival (OS) rate of OSCC only increased from 63 to $65 \%$ in the past 8 years (1-3). Elucidation of the underlying mechanisms of OSCC development may provide promising biomarkers or therapeutic targets, which may lead to improvement of the diagnosis, prognosis and therapy of OSCC.

Gene expression microarray and gene chip are efficient and large-scale detection techniques, which are commonly used to monitor genome-wide expression levels of genes in a given organism or cells, and is particularly suitable for screening differentially expressed genes (DEGs) between two samples (4). Gene chip may provide gene expression profiles in tumour tissues, and these profiles from diverse microarray platforms are submitted to several public databases, including Gene Expression Omnibus (GEO; https://www.ncbi.nlm.nih.gov/geo). Several studies using gene expression microarray and gene chip have been performed on OSCC to explore the DEGs. However, due to sample heterogeneity, there are numerous inconsistencies among the previous studies. Randhawa and Acharya (5) analyzed several gene expression profiles to identify key genes associated with progression of OSCC stages. Zhao et al (6) identified three candidate genes associated with survival of OSCC. Wang et al (7) analyzed two databases to screen out differentially expressed genes of OSCC. However, as the different databases selected in different analysis, the DEGs screened was distinct. Furthermore, the previous studies have not taken ethnic differences into consideration, actually, many studies have proved the ethnic differences may has relevance with disease gene expression profile $(8,9)$. The integration and bioinformatics analysis of multiple expression profiles from different sources may cancel out the differences caused by sample heterogeneity and more reliably predict/confirm universal DEGs associated with OSCC development. 
In the present study, four original gene expression profiles, GSE37991 (10), GSE23558 (11), GSE30784 (12) and GSE56532, were obtained from the GEO database. These four profiles contained a total of 244 OSCC samples and 95 normal oral mucosa samples. The DEGs between OSCC and normal oral mucosa were screened. Gene Ontology (GO) enrichment analysis and Kyoto Encyclopedia of Genes and Genomes (KEGG) enrichment analysis were performed using an online toolset. Subsequently, the protein-protein interaction (PPI) network was established, and the key candidate DEGs were identified and further verified. These key DEGs may serve as potential biomarkers for early diagnosis or as promising targets for the treatment of OSCC.

\section{Materials and methods}

Gene expression profile information and identification of DEGs. The GEO database was searched using the following criteria: Search term, 'OSCC'; study type, 'Expression profiling by array'; publication dates, 2010/1/1-2018/11/21; sample count, >10. Four datasets, GSE37991, GSE30784, GSE23558 and GSE56532, were included in the present analysis. The dataset GSE37991 was based on GPL6883 platform (Illumina HumanRef-8 v3.0 expression beadchip) and included 167 OSCC samples and 45 normal oral mucosa samples (submission year, 2012; year of last update, 2017) (10). The dataset GSE23558 was based on GPL6480 platform and included 27 OSCC samples and 4 oral mucosa samples (submission date, 2010; last update, 2018) (11). The GSE30784 dataset, based on the GPL570 platform (Affymetrix U133 2.0 Plus Gene Chip arrays), included 167 OSCC samples and 45 normal oral mucosa samples (submission date, 2011; last update, 2018) (12). The GSE56532, based on GPL10739, included 10 OSCC samples and 6 normal oral mucosa samples (submission date, 2014; last update, 2014). These 4 gene expression profiles were respectively from different regions, including North America, Australia, China and India, thus averting the differences caused by sample heterogeneity of single profiles and revealing universal DEGs that apply to different ethnic groups, as it has been reported that ethnic difference may affect disease-associated gene expression profiles $(8,9)$. The raw data were downloaded as MINiML files and screened with the limma package in $\mathrm{R}$ (version 3.2.5; https://www.r-project.org/) and subsequently, the $\log _{2}$ of the fold change (logFC) was calculated to screen DEGs between OSCC and normal oral mucosa tissues. $\mid \log \mathrm{FCl}>2$ and adjusted P-value $<0.05$ were set as the cut-off criteria.

Furthermore, the human OSCC data were downloaded from The Cancer Genome Atlas (TCGA) database (https://cancergenome.nih.gov/; accession date, 21 Nov. 2018) and analyzed using UALCAN (http://ualcan.path.uab. edu/index.html) (13).

GO and pathway enrichment analyses of DEGs. The Database for Annotation, Visualization and Integrated Discovery (DAVID; https://david.ncifcrf.gov/home.jsp) is a bioinformatics database that integrates biological data and data mining tools to provide systematic, integrated biometric annotation information for large lists of genes or proteins. GO analysis and KEGG pathway enrichment of DEGs were performed using DAVID to identify the GO terms in the categories biological process, cellular component, molecular function, as well as the signaling pathways of the DEGs involved. $\mathrm{P}<0.05$ was considered to indicate statistical significance.

PPI network construction and identification of candidate genes. The Search Tool for the Retrieval of Interacting Genes and Proteins (STRING; http://string-db.org) is a biological database for searching known and predicted protein-protein interactions (14). In the present study, STRING was used to build PPI networks. The minimum required interaction score was set to medium confidence $(0.4)$ and the organism to Homo sapiens (hsa), and Cytoscape software was then used to visualize the network (15). Cytoscape CentiScape (http://apps.cytoscape.org/apps/centiscape) was used to screen key proteins in the network with a degree centrality of $\geq 4$ set as the criterion.

Validation of the key candidate DEGs. UALCAN (http://ualcan.path.uab.edu/index.html) is used for analyzing cancer transcriptome data. By in-depth analyses of TCGA gene expression data, UALCAN may provide the expression of multiple genes in different tumour types and the association between genes and prognosis (13). The candidate key genes were submitted to the UCLAN database, and the TCGA data (accession date, 21 Nov. 2018) were used to verify the association between the expression of these genes and the prognosis of OSCC.

Expression of plasminogen activator, urokinase (PLAU), integrin subunit $\alpha 3$ (ITGA3) and secreted phosphoprotein 1 (SPP1) in different tumour types. In order to determine whether the key genes identified have any specific roles in OSCC development, the Oncomine database (https://www.oncomine. org) was used to compare the expression of PLAU, ITGA3 and SPP1 in head and neck SCC (HNSCC), head and neck adenoid cystic carcinoma, esophageal SCC and esophageal adenocarcinoma (ACC), respectively. The filters were set as follows: i) Gene: PLAU or SPP1 or ITGA3. ii) Analysis type: Cancer vs. normal. iii) Cancer type: Oral cavity SCC, salivary gland adenoid cystic carcinoma, esophageal SCC or esophageal ACC. iv) Sample type: Clinical specimen. v) Data type: mRNA. vi) Threshold settings: $\mathrm{P}<1 \times 10^{-4}$; FC $>2$; gene rank, top $10 \%$.

SPP1, ITGA3 and PLAU knockdown by lentiviral infection. The lentiviral vectors (Lv)-small hairpin RNA targeting SPP1 (Lv-shSPP1), Lv-shITGA3, Lv-PLAU and negative control (Lv-sh-NC) were purchased from Genechem Co., Ltd. (Genechem Co., Ltd, Shanghai, China). CAL-27 cells were purchased from American Type Culture Collection (Manassas, VA, US) and were cultured in Dulbecco's modified Eagle's medium (Invitrogen; Thermo Fisher Scientific, Inc., Waltham, MA, USA) with $10 \%$ fetal bovine serum (Invitrogen; Thermo Fisher Scientific, Inc.) at $37^{\circ} \mathrm{C}$ with $5 \% \mathrm{CO}_{2}$. Cells were seeded in six-well plates at a density of 50,000 cells/well. After the cells attached for $24 \mathrm{~h}$, the Lv-sh-SPP1, Lv-sh-ITGA3, Lv-sh-PLAU or negative control was added to the cells at a multiplicity of infection of 15 with $5 \mu \mathrm{l}$ polybrene (Sigma-Aldrich; Merck KGaA, Darmstadt, 
Germany) to increase the efficiency of infection. After $96 \mathrm{~h}$, the cells transfected with the Lv vectors were selected with $1 \mu \mathrm{g} / \mathrm{ml}$ puromycin and the transfection efficiency was then observed under a fluorescence microscope to count the rate of green fluorescent protein-positive cells (Supplementary Fig. S1). RNA was extracted and the SPP1, ITGA and PLAU were detected using reverse transcription-quantitative polymerase chain reaction (RT-qPCR) to verify the knockdown of SPP1, ITGA and PLAU.

RNA extraction and RT- $q P C R$. CAL-27 cells with Lv-sh-SPP1, LV-sh-ITGA3, Lv-sh-PLAU, LV-sh-NC or Cal-27 cells without transfection were seeded into 6-well plates at a density of 50,000 cells/well. After 48 h, total RNA was extracted using TRIzol reagent (Invitrogen; Thermo Fisher Scientific, Inc.) and reverse-transcribed into complementary (cDNA) using a RevertAid First Strand cDNA Synthesis kit (Thermo Fisher Scientific, Inc.) according to manufacturer's protocol. Real-time qPCR was performed using a Power SYBR Green PCR Master Mix kit (Applied Biosystems; Thermo Fisher Scientific, Inc.) according to manufacturer's protocol. The primers were as follows: SPP1 forward, 5'-TTCTGATTGGGACAGCCGTG-3' and reverse, 5'-TCTCATCATTGGCTTTCCGCT-3'; ITGA3 forward, 5'-TCAAATGGTTACCCTGTGC-3' and reverse, 5'-ACTGTTTCCTTTCCCTCC-3'; PLAU forward, 5'-CCC CGCTTTAAGATTATTGG-3' and reverse, 5'-CGACCCAGG TAGACGATGTAG-3'. $\beta$-actin was used as the housekeeping reference gene and the primers were as follows: Forward, 5'-CGGGAAATCGTGCGTGAC-3' and reverse 5'-CAGGCA GCTCGTAGCTCTT-3'. The two-step PCR was performed as follows: Pre-denaturation at $95^{\circ} \mathrm{C}$ for $1 \mathrm{~min}$, and 40 cycles of denaturation at $95^{\circ} \mathrm{C}$ for $5 \mathrm{sec}$ followed by annealing/extension at $60^{\circ} \mathrm{C}$ for $20 \mathrm{sec}$. Absorbance values were read at the extension stage. The expression levels were quantified according to the $2^{-\Delta \Delta \mathrm{Cq}}$ method as reported previously (16).

Cell Counting Kit-8(CCK-8) cell proliferation assay. CAL-27 cells transfected with the Lv-sh-NC or CAL-27 cells with knockdown of SPP1, PLAU or ITGA3 were seeded into 96-well plates at 1,500 cells/well and incubated for $0,24,48$ or $72 \mathrm{~h}$. Subsequently, $10 \mu \mathrm{l}$ CCK-8 stain (Biyuntian, Beijing, China) was added to each well. Following incubation for another $2 \mathrm{~h}$ at $37^{\circ} \mathrm{C}$, the absorbance at $450 \mathrm{~nm}$ was determined.

Transwell cell migration and invasion assays. Cell migration and invasion assays were performed in a Transwell plate (Corning Costar, Corning, NY, USA). For the Transwell cell migration assay, CAL-27 cells transfected with the Lv-sh-NC or CAL-27 cells with knockdown of SPP1, PLAU or ITGA3 were seeded at $1 \times 10^{4}$ cells/well in serum-free medium in the upper chambers of the Transwell plate, and the lower chamber contained the culture medium with $10 \%$ fetal bovine serum. The cells were incubated for $8 \mathrm{~h}$ at $37^{\circ} \mathrm{C}$ and subsequently, cells on the top surface of the membrane were wiped off. The cells on the bottom surface of the membrane were then stained with $0.5 \%$ crystal violet (Beijing Solarbio Science \& Technology Co., Ltd., Beijing, China) and examined under a light microscope. Cells from 6 random fields across three replicate wells were counted. The same procedure as for the Transwell migration assay was performed for the Transwell invasion assay, except that the membrane surface in the upper chambers was coated with $20 \mu \mathrm{g}$ ECM gel (cat. no. E1270; Sigma-Aldrich; Merck KGaA).

Statistical analysis. Statistical analysis was performed using SPSS 11.5 for Windows (SPSS, Inc., Chicago, IL, USA). Values are expressed as the mean \pm standard deviation. Differences between multiple groups were analyzed by one-way analysis of variance and Dunnett's t-test. $\mathrm{P}<0.05$ was considered to indicate statistical significance.

\section{Results}

Identification of DEGs in OSCC. The 4 gene expression microarray datasets for OSCC, GSE37991, GSE23558, GSE30784 and GSE56532, were obtained from GEO. By screening of the data with the limma package using $\mathrm{P}<0.05$ and $\mid \log \mathrm{FCl}>2$ as cut-off criteria, 414 DEGs, including 111 upregulated and 303 downregulated genes, were obtained from the GSE37991 expression profile data (Fig. 1A). Furthermore, 1,119 DEGs, including 242 upregulated and 877 downregulated genes, were identified from GSE23558 (Fig. 1B). From GSE30784, 498 DEGs, including 215 upregulated and 283 downregulated genes, were identified (Fig. 1C). In addition, 466 DEGs, including 321 upregulated and 145 downregulated genes, were identified from GSE56532 (Fig. 1D).

After a comprehensive analysis of the 4 datasets, 34 DEGs were identified to be differentially expressed in all of them, among which 14 genes were upregulated and 20 were downregulated in OSCC compared with those in normal oral mucosa tissues (Fig. 2A). Fig. 2B provides a heatmap of the 34 DEGs based on FCs that was generated with R-heatmap software.

GO analysis and signaling pathway enrichment of DEGs in OSCC. The GO analysis of the 34 DEGs was performed using the DAVID database with a significance threshold of $\mathrm{P}<0.05$. The enrichment of the 34 DEGs in GO terms of the categories biological process, cellular component and molecular function was determined. As indicated in Fig. 3A, the major GO terms enriched by the DEGs in the category biological function were cell adhesion, cell proliferation process, chemical homeostasis process, wounding process and immune response process. GO analysis in the category cellular component suggested that the DEGs were mainly accumulated in the extracellular region. The molecular function of the DEGs was mainly associated with cytokine, peptidase and endopeptidase activity. As presented in Fig. 3B, the KEGG pathways enriched by the DEGs were mainly associated with the cytokine-cytokine receptor interaction (hsa04060), Toll-like receptor (TLR) signaling pathway (hsa04620), chemokine signaling pathway (hsa04062), focal adhesion (hsa04510), extracellular matrix-receptor interaction (hsa04512) and phosphoinositide-3 kinase (PI3K)/Akt signaling pathway (hsa04151).

Identification of key candidate DEGs by PPI network analysis. The PPI network of the DEG expression products was constructed using Cytoscape software and the STRING database. In total, 16 DEGs, including 12 upregulated and 

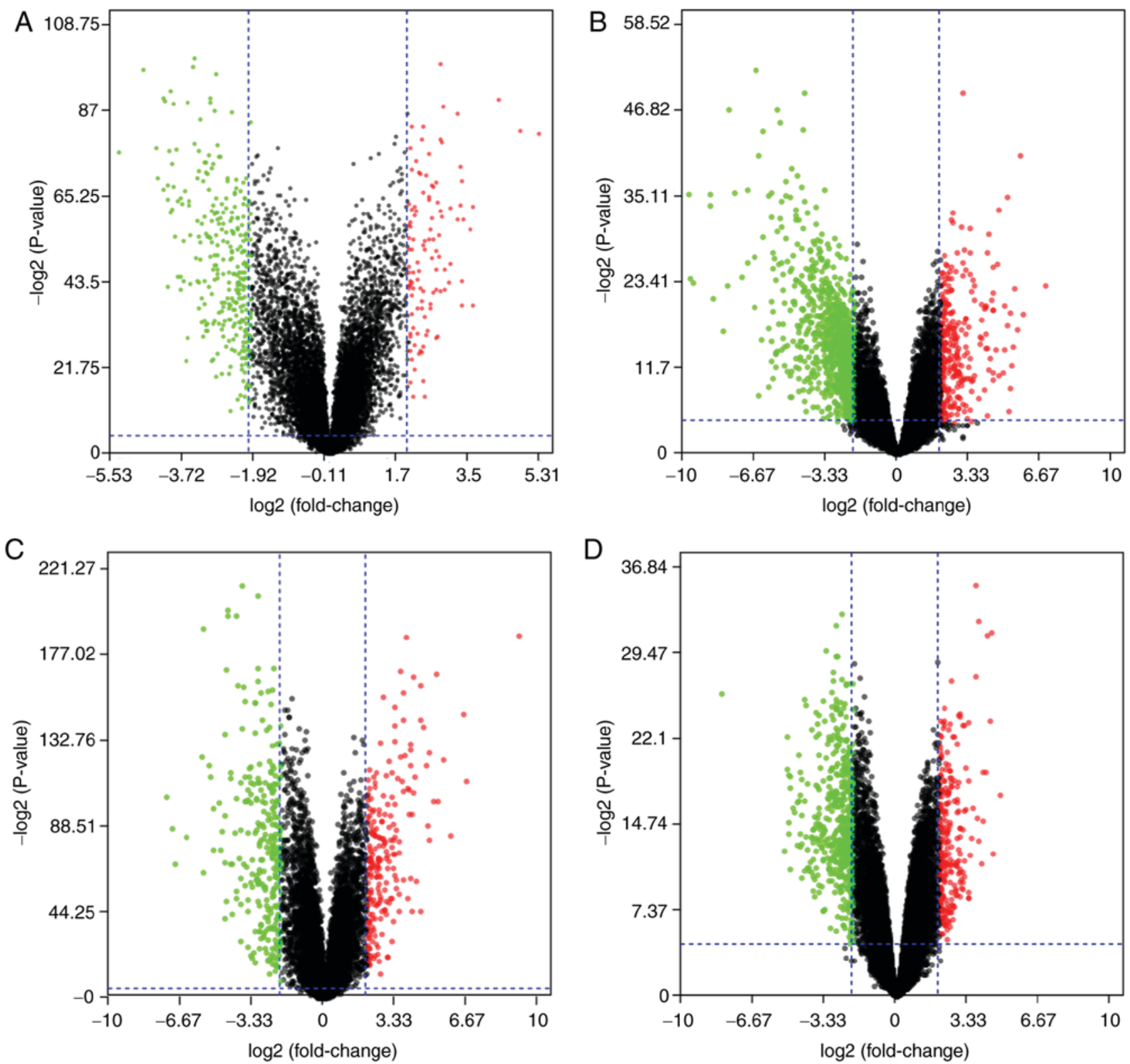

Figure 1. Differential expression of genes between cancerous and non-cancerous samples in the datasets (A) GSE37991, (B) GSE23558, (C) GSE30784 and (D) GSE56532. The red data-points represent upregulated and the green ones represent downregulated genes screened on the basis of Ifold changel $>2.0$ and a corrected P-value of $<0.05$. The black data-points represent genes with no significant difference in expression. The datasets were obtained from the Gene Expression Omnibus database.

4 downregulated genes, were selected for inclusion in the PPI network according to the criteria of 'combined score $>0.5$ ' (Fig. 3C). These 16 DEGs were PLAU, interferon alpha inducible protein 6 (IFI6), C-X-C motif chemokine ligand 9 (CXCL9), C-X-C motif chemokine ligand 11 (CXCL11), Laminin $\gamma 2$ (LAMC2), matrix metallopeptidase (MMP)1, secreted phosphoprotein 1 (SPP1), parathyroid hormone like hormone (PTHLH), keratin 4 (KRT4), serpin family E member 1 (SERPINE1), 2'-5'-oligoadenylate synthetase like (OASL), C-X-C motif chemokine ligand 13 (CXCL13), integrin subunit $\alpha 3$ (ITGA3), matrix metallopeptidase 10 (MMP10), cornulin (CRNN) and transmembrane serine protease 11B (TMPRSS11B). The Cytoscape tool CentiScape was used to screen key genes in the network, with a degree of connectivity of $\geq 4$ as the inclusion criterion. SPP1, SERPINE1,
MMP1, ITGA3 and PLAU were finally selected as candidate key genes.

SPP1, ITGA3 and PLAU are associated with OS of OSCC patients. Patient data from TGCA and were downloaded and subjected to Kaplan-Meier analysis; the log-rank test was applied to determine the influence of the expression status of the candidate key genes on the prognosis of OSCC patients. UALCAN (http://ualcan.path.uab.edu/index.html) was used for analyzing gene expression and patient survival information based on cancer transcriptome data. By in-depth analyses of TCGA gene expression data, UALCAN provided the association between genes and prognosis. SPP1, SERPINE1, MMP1, ITGA3 and PLAU were submitted to the UCLA database and the data were analyzed with the log-rank test. The survival 
A

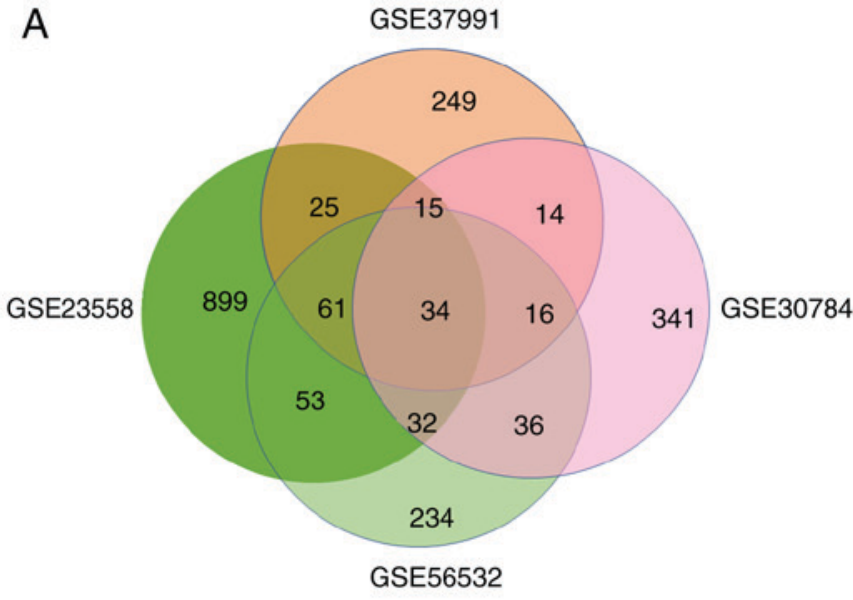

B

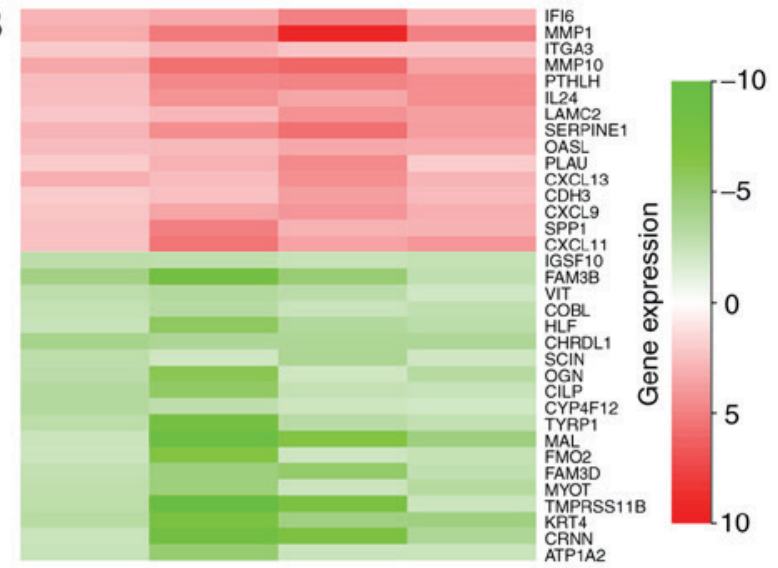

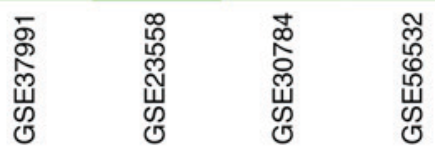

Figure 2. Identification of DEGs. (A) Venn diagram of the DEGs in the 4 datasets. A total of 34 DEGs were included in all 4 data sets. (B) LogFC heatmap image of the 34 commonly changed DEGs of the 4 datasets. The 4 GEO datasets are denoted on the abscissa and the gene names are displayed on the ordinate. Red represents $\log \mathrm{FC}>0$, green represents $\log \mathrm{FC}<0$. DEG, differentially expressed gene; FC, fold change.
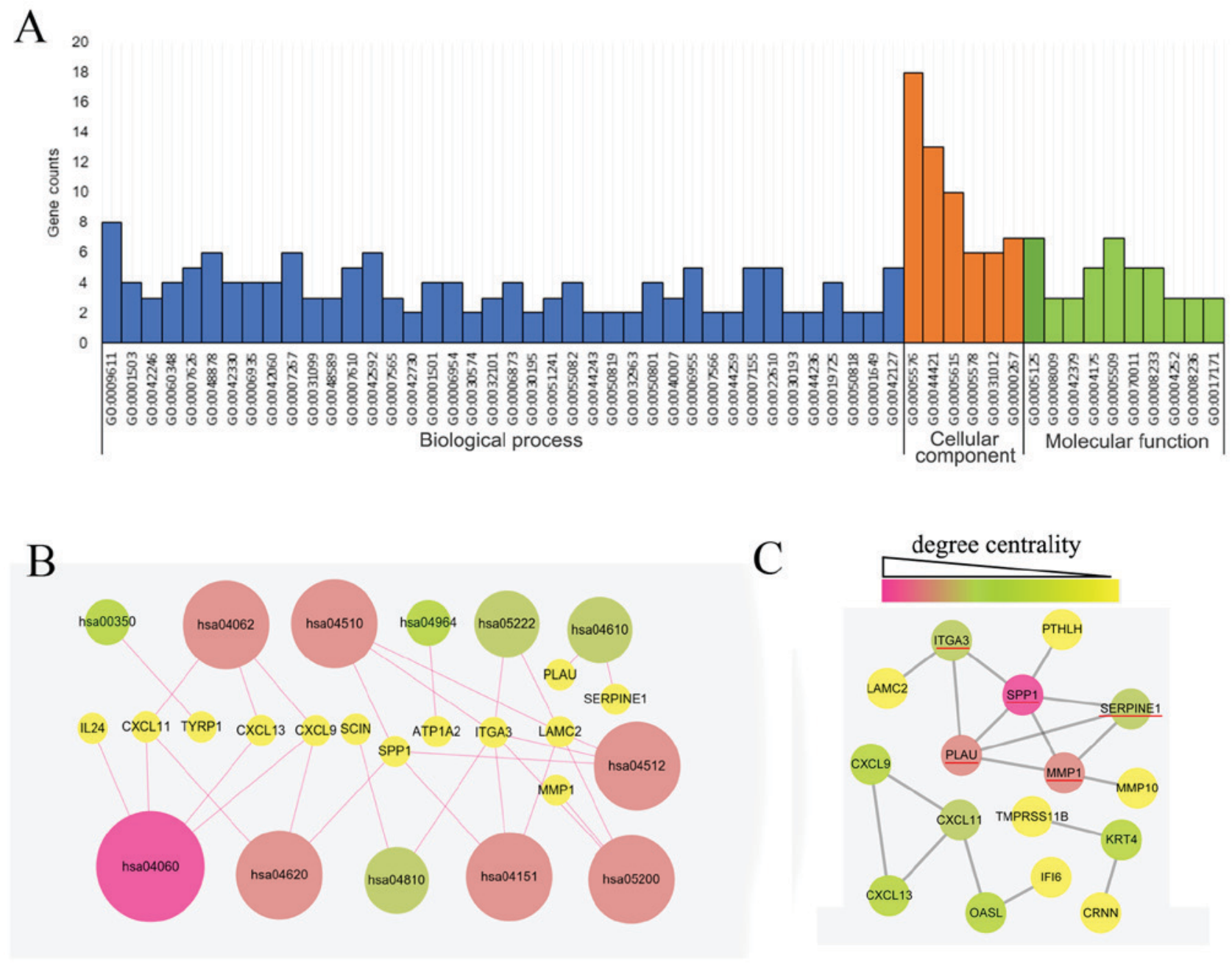

Figure 3. Gene Ontology, KEGG pathway and PPI network analysis of the DEGs in OSCC. (A) Significantly enriched GO terms of DEGs in OSCC. GO analysis of the DEGs in 3 categories (biological process, cellular component and molecular function). (B) Significantly enriched pathway terms of DEGs in OSCC. (C) Complex PPI network and modular analysis. Using the Search Tool for the Retrieval of Interacting Genes online database, a total of 16 DEGs were filtered and included in the PPI network complex. The different colors indicated the different degree centralities. The underlined genes were hub genes identified. GO, Gene Ontology; KEGG, Kyoto Encyclopedia of Genes and Genomes; DEG, differentially expressed gene; PPI, protein-protein interaction; OSCC, oral squamous cell carcinoma. 
analysis based on TCGA data revealed that OSCC patients with high SPP1, ITGA3 or PLAU expression had a shorter OS than those with low/intermediate expression of SPP1, ITGA3 or PLAU (P<0.05; Fig. 4).

Comparison of SPPI, PLAU and ITGA3 expression in database of esophageal and head and neck SCC or ACC. To determine whether SPP1, PLAU or ITGA3 are associated with OSCC development, the expression of these genes was compared in the Oncomine database entries for esophageal and head and neck SCC or ACC. As presented in Fig. 5, ITGA3 was overexpressed in head and neck SCC, esophageal SCC and esophageal ACC samples compared with that in the corresponding para-tumour tissues; however, there were no significant differences in ITGA3 expression between head and neck ACC and the corresponding para-tumour tissues. The expression of PLAU was much higher in head and neck SCC, esophageal SCC and esophageal ACC than that in the corresponding para-tumour tissues, whereas the expression of PLAU in head and neck ACC was not significantly different compared with that in the corresponding para-tumour tissues. Furthermore, the expression of SPP1 in esophageal and head and neck SCC or ACC was upregulated compared with that in the corresponding para-tumour tissues. These results indicated that the association of these genes with the development of OSCC is not specific, as they are also associated with the development of other cancer types.

SPP1, ITGA3 and PLAU are associated with cell proliferation, migration and invasion. As the Bioinformatics analysis revealed that SPP1, PLAU and ITGA3 are associated with the prognosis of OSCC patients, the function of these genes in OSCC was then experimentally confirmed using cell biological techniques. To identify the biological significance of SPP1, ITGA3 and PLAU in the development and progression of OSCC, it was assessed whether these three genes regulate the proliferation, migration and invasion of OSCC cells. Using Lv transfection technique, SPP1, ITGA3 and PLAU were knocked down in CAL-27 cells, a cell line derived from OSCC. The knockdown rates of SPP1, ITGA3 and PLAU were determined to be $44.78,28.17$ and $31.43 \%$, respectively, indicating successful knockdown of the individual genes (Fig. 6A). The CAL-27 cells transfected with Lv-sh-NC, as well as CAL-27 cells with SPP1, ITGA3 and PLAU knockdown were cultured in a 96-well plate for 0, 24, 48 and $72 \mathrm{~h}$, and then subjected to a CCK- 8 cell proliferation assay. As presented in Fig. 6B, CAL-27 cells with knockdown of SPP1 or PLAU, but not ITGA3, possessed a lower capacity for cell proliferation. Furthermore, the migration and invasion ability of CAL-27 cells after knockdown of SPP1, ITGA3 or PLAU, was determined, as presented in Fig. $6 \mathrm{C}$ and D, respectively. The Transwell migration and invasion assays indicated that CAL-27 cells with knockdown of SPP1 or PLAU, but not ITGA3, possessed a decreased capacity for cell migration and invasion. Therefore, the cell proliferation, migration and invasion assays indicated that SPP1 and PLAU may function as oncogenes in OSCC.

\section{Discussion}

OSCC is one of the most common types of HNSCC (1). OSCC is a type of malignant tumour with properties of rapid
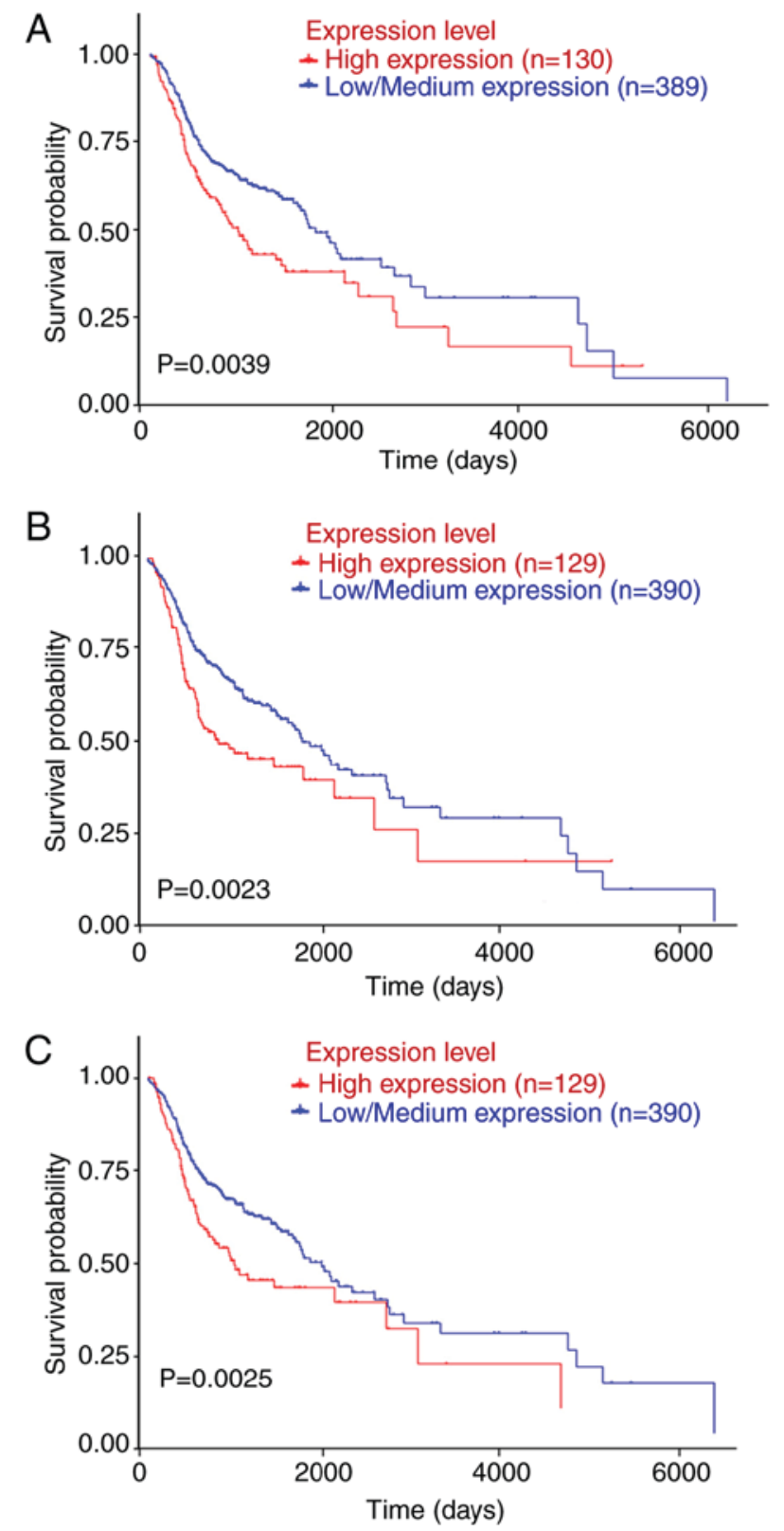

Figure 4. Kaplan-Meier analysis of the association of SPP1, ITGA3 and PLAU with the survival of OSCC patients. Comparison of the overall survival in cases with or without high expression of (A) SPPI, (B) ITGA3 or (C) PLAU. The raw data were extracted from the UALCAN database. SPP1, secreted phosphoprotein 1; ITGA3, integrin subunit $\alpha 3$; PLAU, plasminogen activator, urokinase.

progression, high metastatic capacity and high mortality; however, biomarkers or targets for OSCC diagnosis and therapy are currently insufficient. Searching for genes that are differentially expressed in tumour vs. normal tissues will lead to the further elucidation of the pathogenesis of OSCC and may provide promising biomarkers or targets for early diagnosis and therapy.

In the field of genomics, microarray and high-throughput sequencing technologies are widely used for exploring changes in the genetic aspects of a disease. In recent decades, several microarray and high-throughput sequencing technologies have been used in OSCC; however, the molecular mechanisms and pathogenesis of OSCC remain to be fully elucidated. In the 

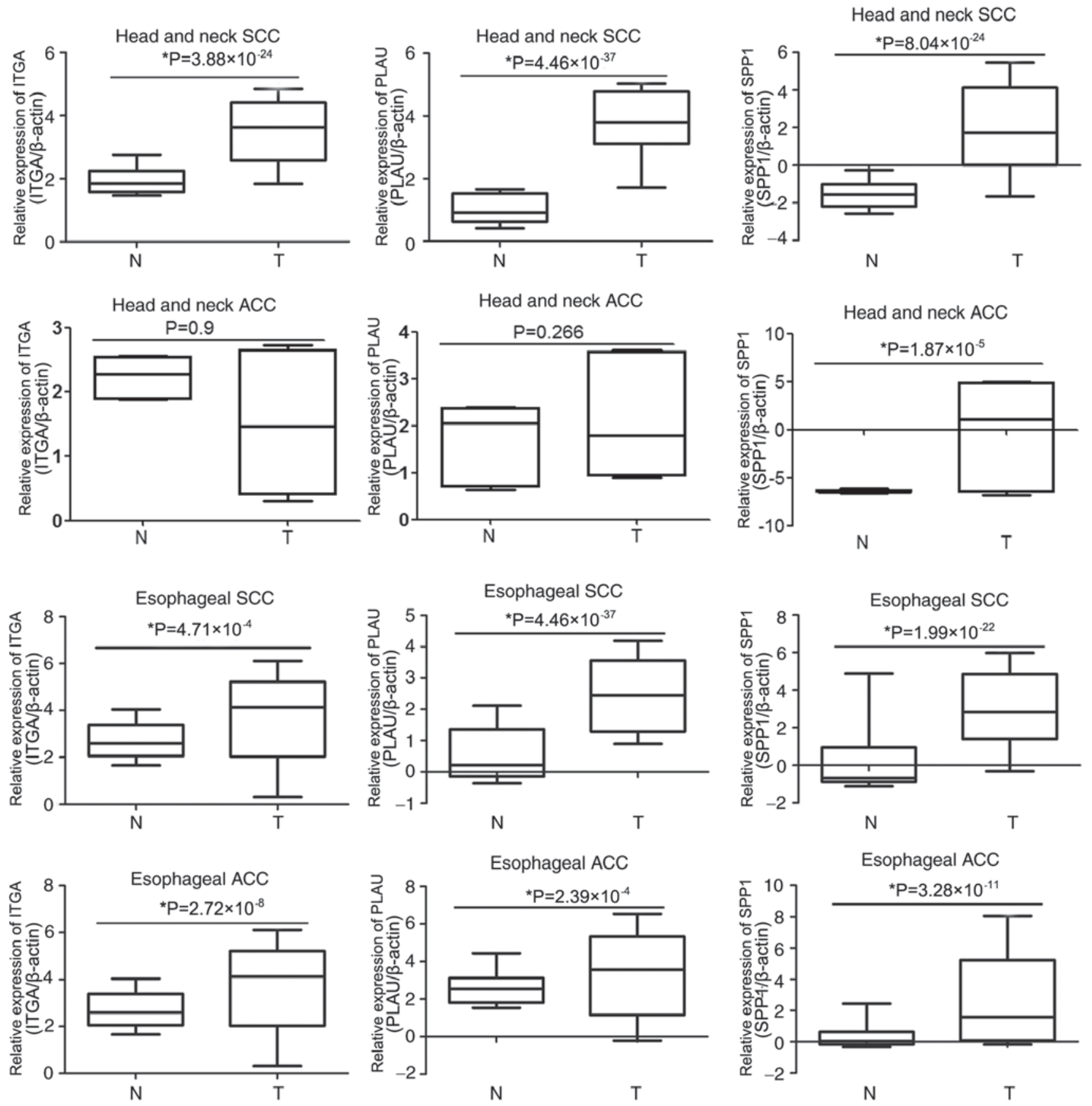

Figure 5. Comparison of SPP1, PLAU and ITGA3 expression in esophageal and head and neck SCC or ACC. The expression of SPP1, PLAU and ITGA3 in tumour tissues and adjacent normal tissues of head and neck SCC, head and neck ACC, esophageal SCC and esophageal ACC were analyzed in the Oncomine database. T, tumour; N, normal tissue adjacent to tumour; SPP1, secreted phosphoprotein 1; ITGA3, integrin subunit $\alpha$ 3; PLAU, plasminogen activator, urokinase; SCC, squamous cell carcinoma; ACC, adenocarcinomas.

present study, four gene expression profiles were integrated and deeply analyzed using Bioinformatics methods. Finally, 34 DEGs were identified, including 14 upregulated and 20 downregulated genes in the first step. The upregulated DEGs were MMP1, PLAU, ITGA3, PTHLH, cadherin 3, SPP1, SERPINE1, IFI6, MMP10, CXCL11, interleukin 24, LAMC2, OASL and CXCL9, and the downregulated DEGs were family with sequence similarity 3 member $\mathrm{B}$, flavin containing monooxygenase 2 , tyrosinase related protein 1 , TMPRSS11B, mal, T cell differentiation protein, osteoglycin, HLF transcription factor, PAR bZIP family member, cartilage intermediate layer protein, KRT4, myotilin, ATPase $\mathrm{Na}+\mathrm{K}+$ transporting subunit alpha 2 , chordin like 1, cordon-bleu WH2 repeat protein, immunoglobulin superfamily member 10 , CRNN, vitrin, family with sequence similarity 3 member D, cytochrome P450 family 4 subfamily F member 12, CXCL13 and scinderin.

These 34 DEGs were then subjected to GO term and KEGG signaling pathway enrichment analysis. In addition, the PPI network was constructed and 5 key candidate DEGs were identified. The GO analysis indicated that the DEGs were mainly involved in immune response, cell adhesion and cell proliferation. The KEGG pathway analysis revealed that the DEGs were mainly associated with cytokine-cytokine 

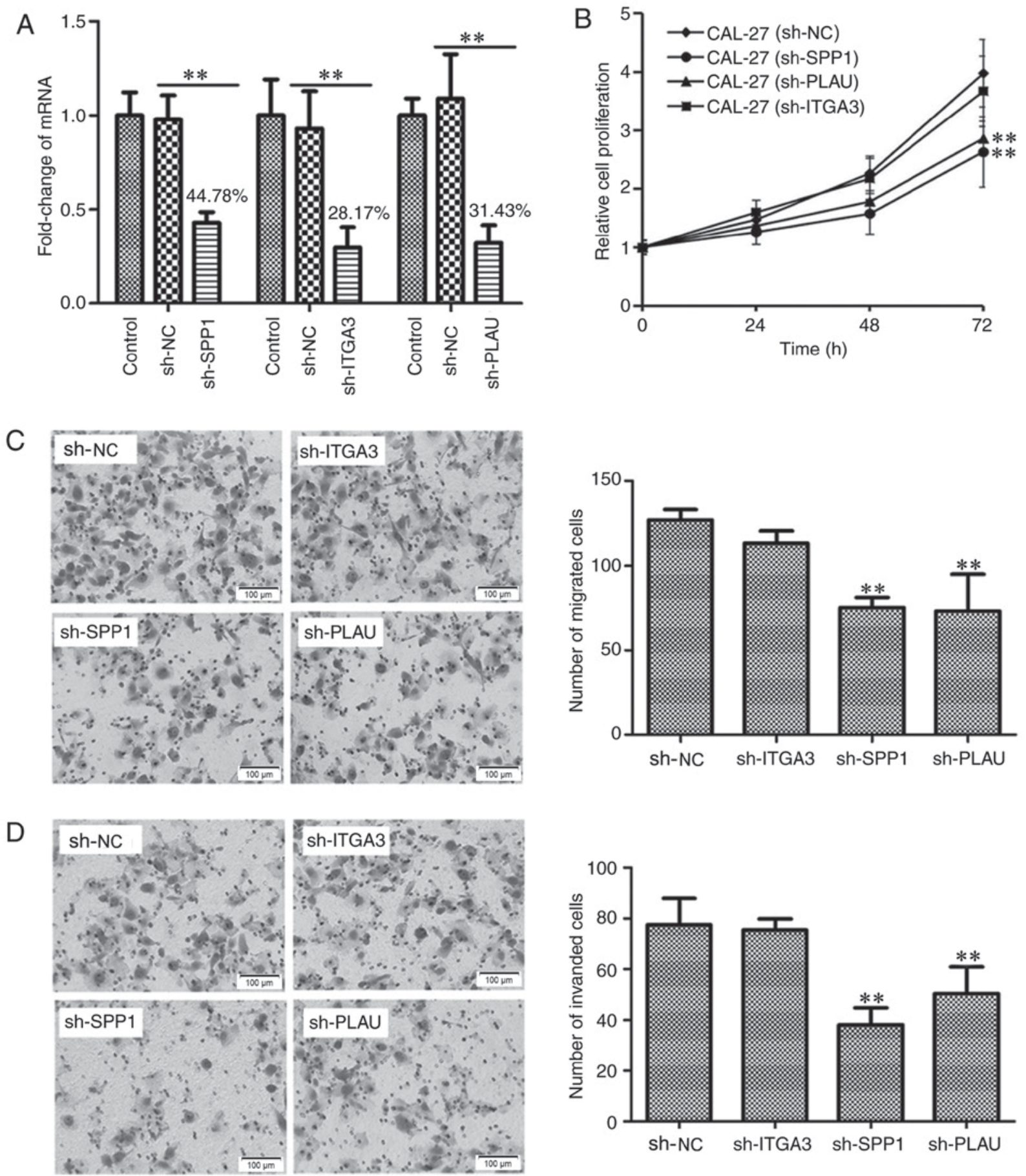

Figure 6. Detection the function of SPP1, ITGA3 and PLAU in regulating OSCC cell proliferation, migration and invasion. (A) Verification of knockdown of SPP1, ITGA3 or PLAU in CAL-27 cells. SPP1, ITGA3 or PLAU were knocked down using a lentiviral transfection technique. Reverse transcriptionquantitative polymerase chain reaction analysis was used to verify the knockdown of SPP1, ITGA3 and PLAU. ** P $<0.01$. (B) Knockdown of SPP1 or PLAU inhibited CAL-27 cell proliferation at $72 \mathrm{~h}$ as indicated by at Cell Counting Kit-8 assay. ${ }^{* *} \mathrm{P}<0.01$ vs. CAL-27 sh-NC group. (C) Knockdown of SPP1 or PLAU inhibited CAL-27 cell migration as indicated by a Transwell migration assay. Photomicrographs of migrated cells are provided in the left panel and quantified migrated cells from 6 separate fields of three replicate wells are provided in the right panel. ${ }^{* *} \mathrm{P}<0.01$ vs. sh-NC group. (D) Knockdown of SPP1 or PLAU inhibited CAL-27 cell invasion. The CAL-27 cells, CAL-27 cell with SPP1, ITGA3 or PLAU knockdown as indicated by a Transwell invasion assay. Photomicrographs of invaded cells are provided in the left panel and quantified invaded cells from 6 separate fields of three replicate wells are provided in the right panel. ${ }^{* *} \mathrm{P}<0.01$ vs. sh-NC group. Values are expressed as the mean \pm standard deviation ( $\mathrm{n}=3$ in A and $\mathrm{n}=6$ in B-D). Groups: Control, empty control with no treatment; sh-NC, CAL-27 cells transfected with negative control lentivirus; sh-SPP1, CAL-27 cells with SPP1 knockdown; sh-PLAU, CAL-27 cells with PLAU knockdown; sh-ITGA3, CAL-27 cells with ITGA3 knockdown. SPP1, secreted phosphoprotein 1; ITGA3, integrin subunit $\alpha$ 3; PLAU, plasminogen activator, urokinase.

receptor interaction, as well as the TLR, chemokine and PI3K/Akt signaling pathways. This was consistent with previous studies, which reported that immune response, cell proliferation and adhesion, and TLR signaling (17), as well as PI3K/Akt signaling (18), have important roles in the genesis and progression of OSCC. 
TLR signaling has been reported to be involved in activating innate and adaptive immune responses and promote cell proliferation, invasion and angiogenesis in a variety of cancer types $(19,20)$. Activation of the TLR signaling pathway promotes malignant cell invasion and the associated metastatic potential by regulating MMPs, interferon-11, p53, PTEN, VEGF, TIMP-1 and integrin/focal adhesion kinase signaling (21-24); therefore, TLR agonists have been suggested to be promising anti-tumour agents (24). In addition, activation of TLR signaling by either extrinsic or intrinsic factors drives tumorigenesis and tumour growth via regulation of a positive feedback loop that amplifies inflammatory and stemnessassociated properties in cancer cells (25). In the present study, 3 candidate genes involved in the TLR signaling pathway were identified, including SPP1, CXCL11 and CXCL9. CXCL9 and CXCL11 belong to the chemokine CXC family, and may be induced by interferon- $\gamma$, which is associated with the regulation of immune cells $(26,27)$. Previous studies have indicated that CXCL9 and CXCL11 were overexpressed and functioned as tumour suppressors in several types of cancer, including ovarian cancer (28), breast cancer (29), lung cancer (30), prostate cancer (27), gastric cancer (31), lymphoma (32) and colorectal cancer (33). Recent studies have also indicated that CXCL9/11 upregulates the expression of PD-L1, suggesting that CXCL9/11 may be factors influencing the effects of immunotherapy (31). However, the biological significance of CXCL9 and CXCL11 in OSCC remains to be fully elucidated; therefore, further study is required to illustrate the role of CXCL9/11 in OSCC development (26).

The PI3K/Akt signaling pathway, which is one of the most frequently dysregulated pathways in cancer, is responsible for several physiological and pathological processes, including cell proliferation, differentiation, apoptosis, angiogenesis and metabolism (34-37). Abnormal activation of PI3K/AKT signaling pathway is associated with the progression and drug resistance of multiple solid tumour types and hematologic malignancies (38-40). It has been reported that the PI3K/Akt signaling pathway regulates the development of tumours by mediating the epithelial-mesenchymal transition directly and by engaging in cross-talk with other signaling pathways (41). In the present study, 3 candidate genes involved in PI3K/Akt signaling pathway, including LAMC2, ITGA3 and SPP1, were identified. LAMC2 is a laminin component and is involved in the development and progression of a variety of tumour types. Huang et al (42) reported that LAMC2 was overexpressed in colorectal cancer and promotes the proliferation and migration of cancer cells; furthermore, LAMC2 was positively correlated with lymph node metastasis and negatively correlated with the survival rate of colorectal cancer patients. In other tumour types, including lung ACC, non-small cell lung cancer, pancreatic ACC and OSCC, the overexpression of LAMC2 was also associated with poor clinical outcome $(43,44)$.

Based on the PPI network and CentiScape, several candidate genes were selected, including SPP1, SERPINE1, MMP1, ITGA3 and PLAU. Furthermore, SPP1, ITGA3 and PLAU were confirmed to be closely associated with the survival rate of OSCC patients. Hubbard et al (45) constructed a mouse model with high expression of SPP1 and revealed that SPP1 induced cell proliferation and differentiation, indicating that SPP1 may have an important role in cancer progression.
Further studies suggested that SPP1 was overexpressed in various types of cancer, including lung ACC, colorectal cancer and ovarian cancer, and significantly associated with the clinicopathological characteristics (46-49). Another study indicated that SPP1 has an important role in mediating macrophage polarization and this may be one of the important mechanisms for its involvement in tumour regulation (50). PLAU has already been reported to be involved in tumour metastasis. Furthermore, PLAU was demonstrated to have an important role in suppressing the function of human and murine regulatory $\mathrm{T}$ cells (51), indicating that PLAU may be associated with the tumour immune escape. ITGA3, a member of the integrin family, has been reported to be aberrantly expressed in numerous types of malignant human tumour, including colorectal cancer, bladder cancer and prostate cancer (52-54).

To further illustrate the roles of SPP1, PLAU and ITGA3 in the genesis and progression of OSCC, the genes were individually knocked down in CAL-27 cells, a cell line of OSCC, and the resultant effect on the cell proliferation, migration and invasion was assessed. It was indicated that SPP1 and PLAU were associated with cell proliferation, migration and invasion, suggesting that SPP1 and PLAU may exert key functions in OSCC. However, ITGA3 was not significantly associated with CAL-27 cell proliferation, migration and invasion, indicating that, at least in CAL-27 cells, ITGA3 does not significantly affect those cell functions; however, whether this is a specific phenomenon in CAL-27 cells or a universal phenomenon in OSCC requires further elucidation.

Of note, a previous Bioinformatics study has determined DEGs in OSCC (5). One discordant point between the present study and the previous one was the datasets selected, as the present study used four datasets from different regions, including North America, Australia, China and India, thus averting the differences caused by sample heterogeneity revealing universal DEGs that apply to different ethnic groups, as it has been reported that ethnic difference may affect disease-associated gene expression profiles $(8,9)$. The other discordant point was that the previous study focused on cancer stage-specific candidate genes, while the present study paid attention to genes associated with the prognosis of OSCC. The previous study screened several genes that were different from those identified in the present study; this may due to the different datasets included and/or the fact that genes associated with the stage of OSCC are different from those associated with prognosis.

In conclusion, the present study identified 34 DEGs from four OSCC datasets using integrated Bioinformatics analysis; these DEGs were significantly enriched in the TLR, chemokine and PI3K/Akt signaling pathways. It was further confirmed that SPP1, ITGA3 and PLAU were closely associated with the survival rate of OSCC patients. Finally, it was experimentally demonstrated that SPP1 and PLAU regulate cell proliferation, migration and invasion of CAL-27 cells. These results may enhance the current understanding of the pathogenesis of OSCC, and these key candidate DEGs may be important biomarkers for early diagnosis and potential targets for the treatment of OSCC. Further studies are required to detect the function of these genes in OSCC and the molecular mechanisms. 


\section{Acknowledgements}

Not applicable.

\section{Funding}

This study was supported by the Medicine and Health Science Technology Development Plan Project of Shandong province (grant no. 2017WSA15041) and the National Natural Science Foundation of China (grant nos. 81472530 and 81602374).

\section{Availability of data and materials}

The datasets generated and/or analyzed during the present study are available in the Figshare repository (https://figshare.

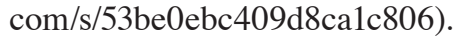

\section{Authors' contributions}

BZ performed Gene Ontology and pathway enrichment analyses of DEGs and drafted the manuscript. JLi analyzed the PPI using STRING and Cytoscape software. KX performed the gene knockdown experiments and reverse transcriptionquantitative polymerase chain reaction. JLiu worked on cell culture. DY performed the Transwell migration and invasion assays. ZM analyzed the association between the candidate genes and prognosis, and as one of the corresponding authors, ZM also participated the design of the study. BZ was the major contributor in designing the present study. All authors read and approved the final manuscript.

\section{Ethics approval and consent to participate}

Not applicable.

\section{Patient consent for publication}

Not applicable.

\section{Competing interests}

The authors declare that they have no competing interests.

\section{References}

1. Siegel RL, Miller KD and Jemal A: Cancer statistics, 2018. CA Cancer J Clin 68: 7-30, 2018.

2. Siegel RL, Miller KD and Jemal A: Cancer statistics, 2016. CA Cancer J Clin 66: 7-30, 2016.

3. Siegel RL, Miller KD and Jemal A: Cancer statistics, 2017. CA Cancer J Clin 67: 7-30, 2017.

4. Petryszak R,Burdett T, Fiorelli B, Fonseca NA, Gonzalez-Porta M, Hastings E, Huber W, Jupp S, Keays M, Kryvych N, et al: Expression atlas update-a database of gene and transcript expression from microarray- and sequencing-based functional genomics experiments. Nucleic Acids Res 42 (Database Issue): D926-D932, 2014.

5. Randhawa V and Acharya V: Integrated network analysis and logistic regression modeling identify stage-specific genes in oral squamous cell carcinoma. BMC Med Genomics 8: 39, 2015.

6. Zhao X, Sun S, Zeng X and Cui L: Expression profiles analysis identifies a novel three-mRNA signature to predict overall survival in oral squamous cell carcinoma. Am J Cancer Res 8: $450-461,2018$
7. Wang Y, Fan H and Zheng L: Biological information analysis of differentially expressed genes in oral squamous cell carcinoma tissues in GEO database. J BUON 23: 1662-1670, 2018.

8. Mitchell KA, Zingone A, Toulabi L, Boeckelman J and Ryan BM: Comparative transcriptome profiling reveals coding and noncoding RNA differences in NSCLC from African Americans and European Americans. Clin Cancer Res 23: 7412-7425, 2017.

9. Hardiman G, Savage SJ, Hazard ES, Wilson RC, Courtney SM, Smith MT, Hollis BW, Halbert CH and Gattoni-Celli S: Systems analysis of the prostate transcriptome in African-American men compared with European-American men. Pharmacogenomics 17: 1129-1143, 2016.

10. Lee CH, Chang JS, Syu SH, Wong TS, Chan JY, Tang YC, Yang ZP, Yang WC, Chen CT, Lu SC, et al: IL-1beta promotes malignant transformation and tumor aggressiveness in oral cancer. J Cell Physiol 230: 875-884, 2015.

11. Ambatipudi S, Gerstung M, Pandey M, Samant T, Patil A, Kane S, Desai RS, Schäffer AA, Beerenwinkel N and Mahimkar MB: Genome-wide expression and copy number analysis identifies driver genes in gingivobuccal cancers. Genes Chromosomes Cancer 51: 161-173, 2012.

12. Chen C, Méndez E, Houck J, Fan W, Lohavanichbutr P, Doody D, Yueh B, Futran ND, Upton M, Farwell DG, et al: Gene expression profiling identifies genes predictive of oral squamous cell carcinoma. Cancer Epidemiol Biomarkers Prev 17: 2152-2162, 2008.

13. Chandrashekar DS, Bashel B, Balasubramanya SAH, Creighton CJ, Ponce-Rodriguez I, Chakravarthi BVSK and Varambally S: UALCAN: A portal for facilitating tumor subgroup gene expression and survival analyses. Neoplasia 19: 649-658, 2017.

14. Franceschini A, Szklarczyk D, Frankild S, Kuhn M, Simonovic M, Roth A, Lin J, Minguez P, Bork P, von Mering C and Jensen LJ: STRING v9.1: Protein-protein interaction networks, with increased coverage and integration. Nucleic Acids Res 41 (Database Issue): D808-D815, 2013.

15. Shannon P, Markiel A, Ozier O, Baliga NS, Wang JT, Ramage D, Amin N, Schwikowski B and Ideker T: Cytoscape: A software environment for integrated models of biomolecular interaction networks. Genome Res 13: 2498-2504, 2003.

16. Livak KJ and Schmittgen TD: Analysis of relative gene expression data using real-time quantitative PCR and the 2(-Delta Delta C(T)) method. Methods 25: 402-408, 2001.

17. Wang L, Zhu R, Huang Z, Li H and Zhu H: Lipopolysaccharide-induced toll-like receptor 4 signaling in cancer cells promotes cell survival and proliferation in hepatocellular carcinoma. Dig Dis Sci 58: 2223-2236, 2013.

18. Koundouros N and Poulogiannis G: Phosphoinositide 3-kinase/Akt signaling and redox metabolism in cancer. Front Oncol 8: 160, 2018.

19. Gay NJ, Symmons MF, Gangloff M and Bryant CE: Assembly and localization of Toll-like receptor signalling complexes. Nat Rev Immunol 14: 546-558, 2014.

20. Shcheblyakov DV, Logunov DY, Tukhvatulin AI, Shmarov MM, Naroditsky BS and Gintsburg AL: Toll-like receptors (TLRs): The role in tumor progression. Acta Naturae 2: 21-29, 2010.

21. Wang W and Wang J: Toll-like receptor 4 (TLR4)/Cyclooxygenase-2 (COX-2) regulates prostate cancer cell proliferation, migration, and invasion by NF-kappaB activation. Med Sci Monit 24: 5588-5597, 2018.

22. Wang F, Jin R, Zou BB, Li L, Cheng FW, Luo X, Geng X and Zhang SQ: Activation of toll-like receptor 7 regulates the expression of IFN- $\lambda 1$, p53, PTEN, VEGF, TIMP-1 and MMP-9 in pancreatic cancer cells. Mol Med Rep 13: 1807-1812, 2016.

23. Lin CH, Lin HH, Kuo CY and Kao SH: Aeroallergen Der p 2 promotes motility of human non-small cell lung cancer cells via toll-like receptor-mediated up-regulation of urokinase-type plasminogen activator and integrin/focal adhesion kinase signaling. Oncotarget 8: 11316-11328, 2017.

24. Braunstein MJ, Kucharczyk J and Adams S: Targeting toll-like receptors for cancer therapy. Target Oncol 13: 583-598, 2018.

25. Yeh DW, Chen YS, Lai CY, Liu YL, Lu CH, Lo JF, Chen L, Hsu LC, Luo Y, Xiang R and Chuang TH: Downregulation of COMMD1 by miR-205 promotes a positive feedback loop for amplifying inflammatory- and stemness-associated properties of cancer cells. Cell Death Differ 23: 841-852, 2016.

26. Tokunaga R, Zhang W, Naseem M, Puccini A, Berger MD, Soni S, McSkane M, Baba H and Lenz HJ: CXCL9, CXCL10, CXCL11/CXCR3 axis for immune activation - A target for novel cancer therapy. Cancer Treat Rev 63: 40-47, 2018. 
27. Tan S, Wang K, Sun F, Li Y and Gao Y: CXCL9 promotes prostate cancer progression through inhibition of cytokines from $\mathrm{T}$ cells. Mol Med Rep 18: 1305-1310, 2018.

28. Bronger H, Singer J, Windmüller C, Reuning U, Zech D, Delbridge C, Dorn J, Kiechle M, Schmalfeldt B, Schmitt M and Avril S: CXCL9 and CXCL10 predict survival and are regulated by cyclooxygenase inhibition in advanced serous ovarian cancer. Br J Cancer 115: 553-563, 2016.

29. Bronger H, Karge A, Dreyer T, Zech D, Kraeft S, Avril S, Kiechle $M$ and Schmitt M: Induction of cathepsin B by the CXCR3 chemokines CXCL9 and CXCL10 in human breast cancer cells. Oncol Lett 13: 4224-4230, 2017.

30. Spaks A: Role of CXC group chemokines in lung cancer development and progression. J Thorac Dis 9 (Suppl 3): S164-S171, 2017.

31. Zhang C, Li Z, Xu L, Che X, Wen T, Fan Y, Li C, Wang S, Cheng Y, Wang X, et al: CXCL9/10/11, a regulator of PD-L1 expression in gastric cancer. BMC Cancer 18: 462, 2018.

32. Ruiduo C, Ying D and Qiwei W: CXCL9 promotes the progression of diffuse large B-cell lymphoma through up-regulating beta-catenin. Biomed Pharmacother 107: 689-695, 2018.

33. Han B, Feng D, Yu X, Liu Y, Yang M, Luo F, Zhou L and Liu F: MicroRNA-144 mediates chronic inflammation and tumorigenesis in colorectal cancer progression via regulating $\mathrm{C}-\mathrm{X}-\mathrm{C}$ motif chemokine ligand 11. Exp Ther Med 16: 1935-1943, 2018.

34. Mayer IA and Arteaga CL: The PI3K/AKT pathway as a target for cancer treatment. Annu Rev Med 67: 11-28, 2016.

35. Spangle JM, Roberts TM and Zhao JJ: The emerging role of PI3K/AKT-mediated epigenetic regulation in cancer. Biochim Biophys Acta Rev Cancer 1868: 123-131, 2017

36. Cao Y, Xia F, Wang P and Gao M: MicroRNA935p promotes the progression of human retinoblastoma by regulating the PTEN/PI3K/AKT signaling pathway. Mol Med Rep 18: 5807-5814, 2018

37. Lunardi A, Webster KA, Papa A, Padmani B, Clohessy JG, Bronson RT and Pandolfi PP: Role of aberrant PI3K pathway activation in gallbladder tumorigenesis. Oncotarget 5: 894-900, 2014.

38. Dong J, Zhai B, Sun W, Hu F, Cheng H and Xu J: Activation of phosphatidylinositol 3-kinase/AKT/snail signaling pathway contributes to epithelial-mesenchymal transition-induced multi-drug resistance to sorafenib in hepatocellular carcinoma cells. PLoS One 12: e0185088, 2017.

39. Xu S, Li Y, Lu Y, Huang J, Ren J, Zhang S, Yin Z, Huang K, Wu G and Yang K: LZTS2 inhibits PI3K/AKT activation and radioresistance in nasopharyngeal carcinoma by interacting with p85. Cancer Lett 420: 38-48, 2018.

40. Xu W, Yang Z, Xie C, Zhu Y, Shu X, Zhang Z, Li N, Chai N, Zhang S, Wu K, et al: PTEN lipid phosphatase inactivation links the hippo and PI3K/Akt pathways to induce gastric tumorigenesis. J Exp Clin Cancer Res 37: 198, 2018.

41. Hamzehzadeh L, Atkin SL, Majeed M, Butler AE and Sahebkar A: The versatile role of curcumin in cancer prevention and treatment: A focus on PI3K/AKT pathway. J Cell Physiol 233: 6530-6537, 2018.

42. Huang D, Du C, Ji D, Xi J and Gu J: Overexpression of LAMC2 predicts poor prognosis in colorectal cancer patients and promotes cancer cell proliferation, migration, and invasion. Tumour Biol 39: 1010428317705849, 2017.
43. Korbakis D, Dimitromanolakis A, Prassas I, Davis GJ, Barber E, Reckamp KL, Blasutig I and Diamandis EP: Serum LAMC2 enhances the prognostic value of a multi-parametric panel in non-small cell lung cancer. Br J Cancer 113: 484-491, 2015.

44. Moon YW, Rao G, Kim JJ, Shim HS, Park KS, An SS, Kim B, Steeg PS, Sarfaraz S, Changwoo Lee L, et al: LAMC2 enhances the metastatic potential of lung adenocarcinoma. Cell Death Differ 22: 1341-1352, 2015.

45. Hubbard NE, Chen QJ, Sickafoose LK, Wood MB, Gregg JP, Abrahamsson NM, Engelberg JA, Walls JE and Borowsky AD: Transgenic mammary epithelial osteopontin (spp1) expression induces proliferation and alveologenesis. Genes Cancer 4: 201-212, 2013.

46. Ng L, Wan T, Chow A, Iyer D, Man J, Chen G, Yau TC, Lo O, Foo CC, Poon JT, et al: Osteopontin overexpression induced tumor progression and chemoresistance to oxaliplatin through induction of stem-like properties in human colorectal cancer. Stem Cells Int 2015: 247892, 2015.

47. Choe EK, Yi JW, Chai YJ and Park KJ: Upregulation of the adipokine genes ADIPOR1 and SPP1 is related to poor survival outcomes in colorectal cancer. J Surg Oncol 117: 1833-1840, 2018.

48. Zeng B, Zhou M, Wu H and Xiong Z: SPP1 promotes ovarian cancer progression via Integrin $\beta 1 /$ FAK/AKT signaling pathway. Onco Targets Therapy 11: 1333-1343, 2018.

49. Zhang W, Fan J, Chen Q, Lei C, Qiao B and Liu Q: SPP1 and AGER as potential prognostic biomarkers for lung adenocarcinoma. Oncol Lett 15: 7028-7036, 2018.

50. Zhang Y, Du W, Chen Z and Xiang C: Upregulation of PD-L1 by SPP1 mediates macrophage polarization and facilitates immune escape in lung adenocarcinoma. Exp Cell Res 359: 449-457, 2017.

51. He F, Chen H, Probst-Kepper M, Geffers R, Eifes S, Del Sol A, Schughart K, Zeng AP and Balling R: PLAU inferred from a correlation network is critical for suppressor function of regulatory T cells. Mol Syst Biol 8: 624, 2012.

52. Kurozumi A, Goto Y, Matsushita R, Fukumoto I, Kato M, Nishikawa R, Sakamoto S, Enokida H, Nakagawa M, Ichikawa T and Seki N: Tumor-suppressive microRNA-223 inhibits cancer cell migration and invasion by targeting ITGA3/ITGB1 signaling in prostate cancer. Cancer Sci 107: 84-94, 2016.

53. Sakaguchi T, Yoshino H, Yonemori M, Miyamoto K, Sugita S, Matsushita R, Itesako T, Tatarano S, Nakagawa M and Enokida H: Regulation of ITGA3 by the dual-stranded microRNA-199 family as a potential prognostic marker in bladder cancer. $\mathrm{Br}$ J Cancer 116: 1077-1087, 2017.

54. Sa KD, Zhang X, Li XF, Gu ZP, Yang AG, Zhang R, Li JP and Sun JY: A miR-124/ITGA3 axis contributes to colorectal cancer metastasis by regulating anoikis susceptibility. Biochem Biophys Res Commun 501: 758-764, 2018.

This work is licensed under a Creative Commons Attribution-NonCommercial-NoDerivatives 4.0 International (CC BY-NC-ND 4.0) License. 